\title{
The Impacts of Complex Social, Environmental, and Behavioral Factors on Obesity
}

\author{
Hammad Akram ${ }^{1 *}$, Gohar Ashraf ${ }^{2}$, Muhammad Asif Ijaz' \\ ${ }^{1}$ Graduate Hamdard College of Medicine, Pakistan \\ ${ }^{2}$ University of Pittsburgh Medical Center, PA, USA
}

\author{
*Correspondence to \\ Hammad Akram \\ Email: \\ hammadakram77@yahoo.com
}

Received September 29, 2017 Accepted August 8, 2018 Published online September 30, 2018

\begin{abstract}
Obesity is a prominent global concern, which is correlated with several chronic diseases and associated mortalities. Social determinants and environmental factors play an important role in the adoption of certain behaviors that cause obesity and related health issues. This makes obesity a complex public health issue dependent on several physiological, pathobiological, and psychological phenomena. Here we aimed to review the complex interrelationship between the social determinants, behavioral factors, and obesity. The literature search was carried out in PubMed, Web of Science, and Embase databases using keywords of "obesity" and/or "multimorbidity" and/or "chronic diseases" along with "social factors", "social determinants", "social determinants of health", "cultural factors", and "Environmental factors". We found the importance of school-based programs in prevention of obesity through behavioral modification. Educational programs and incentives and their impact on obesity and diabetes at the community level were demonstrated. Social factors and health behaviors significantly predicted body mass index (BMI) with gender-specific variations. Furthermore, psychological, emotional, and social experiences of the individuals with obesity had a drastic effect on their mental and physical health. It is apparent that the social factors influence the relations between BMI and weight-related behaviors and outcomes. To understand the mechanisms behind obesity, both quantitative and qualitative methods should be applied in order to examine the overt as well as cognitive aspects of the complex relationships described here.

Keywords: Obesity, Obesogenic factors, Social determinants, Social environment, Multimorbidity, Obesity prevention
\end{abstract}

Please cite this article as follows: Akram $\mathrm{H}$, Ashraf G, ljaz MA. The impacts of complex social, environmental, and behavioral factors on obesity. Int J Basic Sci Med. 2018;3(3):9498. doi:10.15171/ ijbms.2018.17.

\section{Introduction}

Obesity is the prominent global phenomenon of this century and is associated with morbidity and mortality of chronic diseases and their risk factors. ${ }^{1}$ According to the World Health Organization (WHO), the global prevalence of overweight and obesity in adults has increased around $8 \%$ between the years 1980 and 2013 . $^{2}$ The role of obesity in the etiology of several chronic diseases and related mortality has been well documented. ${ }^{3-5}$ Obesity has reached epidemic levels in developed as well as developing countries and is known to have a significant impact on both physical and emotional health. High sugar intake, increased portion size, insufficient physical activity, and more screen time have contributed to increased obesity. ${ }^{6}$
Social determinants and environmental factors could play an important role among humans in the adoption of certain behaviors leading to obesity and related health issues. ${ }^{7,8}$ Factors such as excessive food intake, lack of or insufficient physical activity, inadequate sleep, certain drugs, and some environmental factors that can lead to obesity are denoted as "obesogenic factors" and have an evident impact on the outcomes of chronic diseases. ${ }^{3-9}$

This review aimed to explore the complex interrelationships between the social environment, social determinants, and behavioral factors and their role in the development of obesity and its adverse health outcomes. Furthermore, we described a few examples of different public health interventions and their impact on

(C) 2018 The Author(s); Published by Zabol University of Medical Sciences. This is an open-access article distributed under the terms of the Creative Commons Attribution License (http://creativecommons.org/licenses/by/4.0), which permits unrestricted use, distribution, and reproduction in any medium, provided the original work is properly cited. 
behavioral modifications.

\section{Materials and Methods}

The literature search was carried out using keywords and Medical Subject Headings (MeSH) terms in the English language. The search was executed on PubMed, Web of Science, and Embase databases. The used terms were "obesity" and/or "multimorbidity" and/or "chronic diseases" along with "social factors", "social determinants", "social determinants of health", "cultural factors", and "environmental factors". The phrases such as "obesity and social determinants of health" were also searched. Boolean operators (AND, OR) were used to make different combinations of search terms. An attempt was made to select these keywords as per accordance to the population, exposure, outcome (PEO) framework, but most of the searches were only based on exposure (e.g. social determinants, environment) and outcome of interest. The articles concerned with the impacts of social and environmental factors on obesogenic behavior (such as types and availability of diet, physical activity opportunities, and socioeconomic status) were also reviewed. The articles encompassing clinical and pathobiological aspects of obesity were excluded from the literature review.

The screening of articles was carried out by reviewing their abstracts. Attention was given to the background, objectives, and methods of the abstracts before moving forward to review the complete article. Article titles representing pharmaceutical, biochemical, pathobiological, and occasionally clinical aspects of the issue were not considered in the literature review. The articles with the titles containing "national survey", "cross-sectional", "population-based", "social factors", "environment", and "cultural factors" were considered for further review. Reference lists of the selected articles were also examined to identify relevant papers. The primary search resulted in 748210 items which were reduced to 44 articles after applying different combinations of keywords, phrases, and filters. Different types of research designs (e.g. exploratory, descriptive, explanatory) were considered for review.

\section{Results}

School-Based Interventions

The article by Veugelers and Fitzgerald evaluated the effectiveness of school-based programs in the prevention of obesity among children. ${ }^{10}$ This is important as obesity and certain health behaviors during childhood could lead to adulthood obesity and related complications. The authors compared the effectiveness of schoolbased programs targeting obesity and overweight, diet quality and physical activity and compared the results with the conditions without such programs. The aim of authors in this study was to examine the relationship between school-based programs and their role in obesity prevention. The primary objectives were to assess the diet, physical activity, and obesity among schools with 1) General nutritional programs, 2) Coordinated program known as "Annapolis Valley Health Promoting Schools (AVHPSP)" that targeted eating practices and physical activity, and 3) No nutritional programs. The authors evaluated these programs and studied their effectiveness by assessing behavioral outcomes. The results of this study showed that the students attending schools with a coordinated program (AVHPSP) had better rates in term of overweight and obesity, diet quality, fruit and vegetable consumption, fat consumption, physical activity, and sedentary activities. The statistically significant favorable results were obtained for the students in the areas of overweight and obesity, diet quality, fruit and vegetable consumption in AVHPSP-implemented schools compared to the students of schools without a nutritional program. The results between schools with and without nutritional programs were not much different except that there were slightly lower rates of overweight and obesity among the students who attended schools with a nutritional program, though this difference was not statistically significant. This study described how interventions can play an important role in younger populations. A study based on the European School Fruit and Vegetable Scheme with a sample size of 702 and age range of 7-10 years (thirdfourth grades) showed that parental modeling and peer influence had a significant positive impact on fruit and vegetable intake while verbal directive had a negative impact. ${ }^{11}$ Furthermore, preference and knowledge about different types of fruits and vegetables had a significant positive impact on their intake.

Socio-economic, Behavioral, and Environmental Influences Ludwig et al evaluated the effect of a social experiment using incentives on the obesity and diabetes among the samples. ${ }^{12}$ The authors implemented this study based on the theory that neighborhood situations such as poverty and racial disparities may increase the risk of obesity and diabetes among deprived individuals. Using an experimental approach, they attempted to explain the changes in circumstances and their role in selected outcomes. The participants from high-poverty areas were randomly assigned to three groups. One group received counseling and low-poverty vouchers to support relocation to a low poverty rate census area. The second group was given a standard voucher without any counseling. The third or control group received no intervention or extra incentives. The authors found that after a long-term follow-up, the intervention group who received low poverty vouchers had a lower prevalence of obesity and glycated hemoglobin levels (diabetes) compared to the controls. No significant differences were identified between standard vouchers group and controls. The individuals who spent more time in the low-poverty area had positive changes in their diabetes and obesity 
outcomes when the results were examined using a doseresponse model. Such result was also supported by an Iranian population-based cross-sectional study in which higher socioeconomic status was associated with lower rate of obesity. ${ }^{13}$ Furthermore, as evident from the results of two different longitudinal and cohort studies, socioeconomic disadvantages and deprived neighborhood increased the risk of multimorbidity (two or more chronic health problems) and higher body mass index (BMI) among participants, respectively. ${ }^{14,15}$ In a systemic review, the deprived neighborhoods were found to have limited access to supermarkets. Moreover, access to the takeaway outlets had a relationship with an increased body weight while the opposite was seen in the areas with better access to the supermarkets. ${ }^{8}$ In the latter situation (good access to the supermarkets), the low obesity rates could be attributed to the access to fresh fruits, vegetables and healthier food options usually available at these supermarkets.

Ball et al in their study aimed to examine the role of health behaviors in explaining the relationships between social factors and obesity. ${ }^{16}$ The authors used a sample of 8667 adults who participated in the 1995 Australian National Health and Nutrition Survey, which collected data pertaining to the health factors, including objectively measured height and weight, health behaviors, and social factors such as family status, employment status, housing situation, and migration status. The authors selected behaviors such as physical activity, alcohol use, diet, and weight control efforts, and examined their impacts on social group's differences in obesity. In this study, a model of these components (i.e. social factors based on employment, housing, migration, family unit, behaviors, and BMI) was tested. The authors used a nonexperimental, descriptive study design for which the data were previously collected through a cross-sectional survey. The authors found that in the adjusted analytical model, the social factors significantly predicted BMI with gender-specific variations. Furthermore, behavioral and social factors interacted with each other depending on the gender to predict BMI. The men living in the lower levels of housing (rental properties or houses with fewer number of bedrooms) and family status (familial marriages) had higher BMI. The women with part-time jobs and lower occupation status (unemployed or receiving pension or benefits) had higher BMI compared with the women with full-time jobs or high-level occupations (including managerial or professional positions).

\section{Psychological and Social Influences (Qualitative)}

The study by Rand et al explored psychological, emotional, and social experiences of individuals with obesity. ${ }^{17}$ To understand these experiences, the authors used a qualitative approach for which 4 levels of the social-ecological model (SEM) derived from the mental well-being of obese individuals were employed. At an individual level, they examined food as a coping mechanism and source of emotional distress. At the interpersonal level, they experienced blame and shame by family and friends regarding their body weight plus lack of support from health professionals. At the organizational level, the participants experienced insufficient mental health support in obesity management programs. Finally, at the community level, obese individuals faced negative mental well-being impacts of the social stigma of obesity. The authors used a methodology that included interactions between the researchers and participants and implemented qualitative semi-structured interviews and used transcripts from 19 obese participants and 16 health professionals. Furthermore, two frameworks were applied to collect relevant data for this study (i.e. WHO domains of mental well-being and SEM levels which were aligned according to the identified mental well-being themes). The results showed that at "individual level", about half of the respondents considered food as a coping mechanism and a source of emotional distress having a negative influence on mental well-being. At "interpersonal level", the expression of blame and shame by family, friends, and healthcare providers had a detrimental impact on the psychological and mental health as well as on losing body weight. At "organization level", the participants felt the need for judgment-free support programs to address their psychological issues pertaining to the obesity. At "community level", the individuals reported to experience the stigma of obesity in verbal and non-verbal manners.

\section{Summary}

Social, cultural, environmental, and behavioral factors are interconnected in influencing obesity and its underlying processes. Along with the general or occult comprehension of obesity and related outcomes, understanding the psychological aspects of why society or individuals adopt certain characteristics such as obesity is equally crucial. Furthermore, examination of psychological effects of obesity could be useful in formulating preventive programs. It is important to understand individuals' perceptions of what constitutes a healthy diet and their impression of what is the health status among children, adolescents, and adults. This could be coupled with different surveillance approaches (e.g. ongoing school-based programs to monitor body weight, physical activity, nutritional habits, workplace wellness programs and hospital-based registries) to further explore obesity and its risk factors. Studies have indicated that sedentary lifestyles, especially long sitting hours at work, sleep hygiene, and environmental factors such as air and weather pollution act under the concept of the epidemiological triad (host, agent, and environment) to influence obesity (Figure 1). ${ }^{18,19}$ Sleep duration has been known to be associated with weight-related changes; for example, a review study indicated that sleeping less could be associated with a higher risk of obesity among 


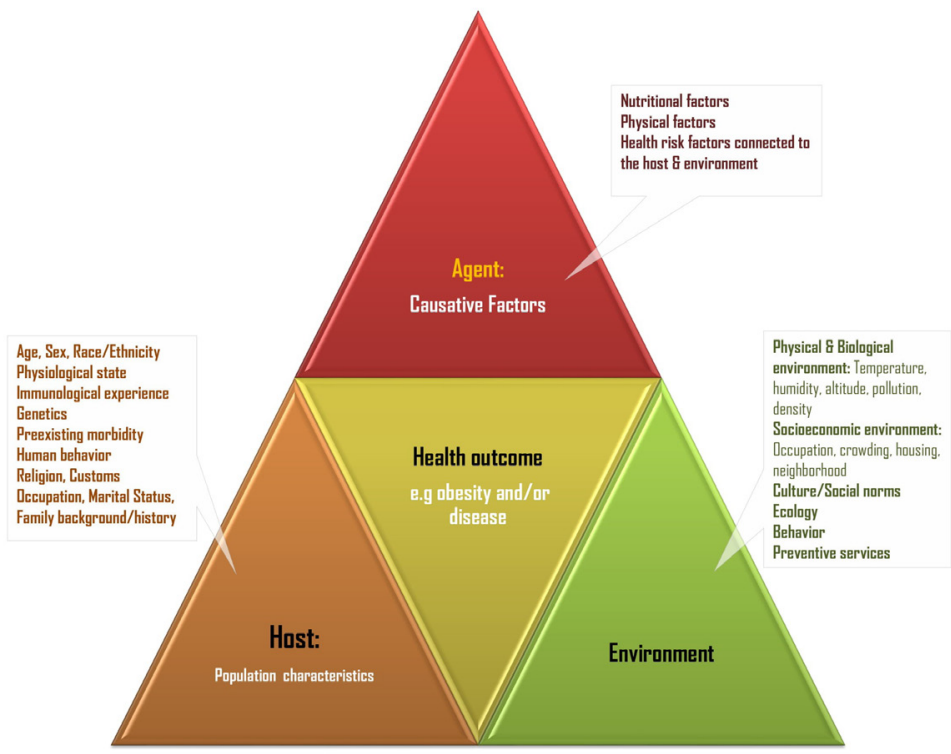

Figure 1. Epidemiological Triad Example of Obesity and Related Factors.

children and young adults. The lack of sleep can be due to the host-related as well as environmental factors. ${ }^{20}$

\section{Conclusion}

The present study reviewed the impacts of some of the social, environmental, and economic factors on obesity and related items. It is apparent that the social factors influence the relation between BMI and weight-related behaviors and outcomes. To understand the mechanisms behind obesity, both quantitative and qualitative methods are essential to examine the overt as well as cognitive aspects of the complex relationships between obesity and risk-factors. While health promotion activities targeting diet, physical activity, and general awareness are important, interventions incorporating psychological support are also critical. In addition, interventions targeting younger populations and incorporating multidimensional approaches seem to have a better effect on the positive and long-term behavioral changes.

\section{Ethical Approval}

Not applicable.

\section{Competing Interests}

Authors declare that they have no competing interests.

\section{References}

1. Murray CJ, Lopez AD. Mortality by cause for eight regions of the world: Global Burden of Disease Study. Lancet. 1997;349(9061):1269-1276. doi:10.1016/s01406736(96)07493-4

2. $\mathrm{Ng} \mathrm{M}$, Fleming $\mathrm{T}$, Robinson $\mathrm{M}$, et al. Global, regional, and national prevalence of overweight and obesity in children and adults during 1980-2013: a systematic analysis for the Global Burden of Disease Study 2013. Lancet. 2014;384(9945):766-781. doi:10.1016/s0140-

\section{6(14)60460-8}

3. Wang YC, McPherson K, Marsh T, Gortmaker SL, Brown $\mathrm{M}$. Health and economic burden of the projected obesity trends in the USA and the UK. Lancet. 2011;378(9793):815825. doi:10.1016/s0140-6736(11)60814-3

4. Calle EE, Thun MJ. Obesity and cancer. Oncogene. 2004;23(38):6365-6378. doi:10.1038/sj.onc. 1207751

5. Manson JE, Colditz GA, Stampfer MJ, et al. A prospective study of obesity and risk of coronary heart disease in women. N Engl J Med. 1990;322(13):882-889. doi:10.1056/ nejm199003293221303

6. Rosiek A, Maciejewska NF, Leksowski K, RosiekKryszewska A, Leksowski L. Effect of Television on Obesity and Excess of Weight and Consequences of Health. Int J Environ Res Public Health. 2015;12(8):9408-9426. doi:10.3390/ijerph120809408

7. Bennett GG, Wolin KY, Duncan DT. Social Determinants of Obesity. Obesity Epidemiology: Methods and Applications. Edited by: Hu FB. 2008.

8. Giskes K, van Lenthe F, Avendano-Pabon M, Brug J. A systematic review of environmental factors and obesogenic dietary intakes among adults: are we getting closer to understanding obesogenic environments? Obes Rev. 2011;12(5):e95-e106. doi:10.1111/j.1467789X.2010.00769.x

9. Obesogenic. (n.d.) Collins Dictionary of Medicine. (2004, 2005). https://medical-dictionary.thefreedictionary.com/ obesogenic. Accessed January 7, 2018.

10. Veugelers PJ, Fitzgerald AL. Effectiveness of school programs in preventing childhood obesity: a multilevel comparison. Am J Public Health. 2005;95(3):432-435. doi:10.2105/ajph.2004.045898

11. Hass J, Hartmann M. What determines the fruit and vegetables intake of primary school children? - An analysis of personal and social determinants. Appetite. 2018;120:8291. doi:10.1016/j.appet.2017.08.017

12. Ludwig J, Sanbonmatsu L, Gennetian L, et al. Neighborhoods, obesity, and diabetes--a randomized 
social experiment. N Engl J Med. 2011;365(16):1509-1519. doi:10.1056/NEJMsa1103216

13. Emamian $\mathrm{MH}$, Fateh $\mathrm{M}$, Hosseinpoor $\mathrm{AR}$, Alami A, Fotouhi A. Obesity and its socioeconomic determinants in Iran. Econ Hum Biol. 2017;26:144-150. doi:10.1016/j. ehb.2017.03.005

14. Katikireddi SV, Skivington K, Leyland AH, Hunt K, Mercer SW. The contribution of risk factors to socioeconomic inequalities in multimorbidity across the lifecourse: a longitudinal analysis of the Twenty-07 cohort. BMC Med. 2017;15(1):152. doi:10.1186/s12916-017-0913-6

15. Owen G, Jones K, Harris R. Does neighbourhood deprivation affect the genetic influence on body mass? Soc Sci Med. 2017;185:38-45. doi:10.1016/j. socscimed.2017.05.041

16. Ball K, Mishra GD, Crawford D. Social factors and obesity: an investigation of the role of health behaviours. Int J Obes Relat Metab Disord. 2003;27(3):394-403. doi:10.1038/ sj.ijo.0802237

17. Rand $\mathrm{K}$, Vallis M, Aston M, et al. "It is not the diet; it is the mental part we need help with." A multilevel analysis of psychological, emotional, and social well-being in obesity. Int J Qual Stud Health Well-being. 2017;12(1):1306421. do i:10.1080/17482631.2017.1306421

18. Nurse Key. Epidemiology. In: Epidemiological Triangle. https://nursekey.com/5-epidemiology/.

19. Centers for Disease Control and Prevention. Principles of Epidemiology in Public Health Practice, Third Edition. An Introduction to Applied Epidemiology and Biostatistics. Lesson 1: Introduction to Epidemiology. https://www.cdc. gov/ophss/csels/dsepd/ss1978/lesson1/section8.html.

20. Nielsen LS, Danielsen KV, Sorensen TI. Short sleep duration as a possible cause of obesity: critical analysis of the epidemiological evidence. Obes Rev. 2011;12(2):78-92. doi:10.1111/j.1467-789X.2010.00724.x 